\title{
On the timing of the next great solar activity minimum
}

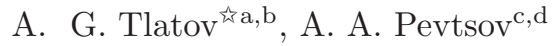 \\ ${ }^{a}$ Kislovodsk Mountain Astronomical Station of Pulkovo Observatory, Kislovodsk, Russia \\ ${ }^{b}$ Kalmyk State University, Elista, Russia \\ ${ }^{c}$ National Solar Observatory, Sunspot, NM 88349, U.S.A. \\ ${ }^{d}$ ReSoLVE Centre of Excellence, Space Climate research unit, University of Oulu, PO Box \\ 3000, 90014 Oulu, Finland
}

\begin{abstract}
The long-term variations in solar activity are studied using the dataset comprised of sunspot number and ${ }^{14} C$ radioisotope timeseries. We use a novel $S_{200}$ index to identify possible past Grand Minima (GM). The Maunder, Oort, Wolf and Spörer Minima fall in phase with the minimum of $S_{200}$ index. We also show GM develop in clusters, with a separation of about 400-600 years between individual GM. Extending these found similarities to modern solar activity, it is predicted that next grand solar minimum may occur in about $\sim 2090 \pm 20$.
\end{abstract}

Keywords: Sun, Solar cycle, long-term prediction

2010 MSC: 00-01, 99-00

\section{Introduction}

One of the main questions in the physics of solar periodicity is the prediction of activity for the next decade. The modern era, especially the activity in the 20th century, has been characterized by high solar activity. However, there are indications this might be changing. The 22nd and 23rd solar cycles defied the Gnevyshev-Ohl (G-O) rule, which otherwise had been satisfied for approximately 150 years, starting with the 10th solar cycle. Following them,

\footnotetext{
${ }^{*}$ Corresponding author

Email addresses: tlatov@mail.ru; tel: +79282522125; fax: +78793731088; ( A. G. Tlatov ${ }^{\text {tr }}$ ), apevtsov@nso.edu (A. A. Pevtsov)
}

Preprint submitted to Journal of ${ }^{A} T_{E} X$ Templates

April 6, 2017 
the 24th cycle preceded one of the lowest known minima of activity. The development of the 24th cycle testifies to its relatively low activity. Based on international sunspot number time series, by its shape, the cycle 24 is similar to cycle 14 (1902-1913). By its amplitude, however, it is slightly lower than cycle 14, which makes it the weakest cycle in the last 115 years. These facts might indicate impending change in the behavior of the solar cycle, a change which may also occurred in the past [1]. A good prediction of future solar activity should address the following questions: Is a change in the behavior of the solar cycle currently occurring, and how quickly can this change transpire. The answers to these questions may be provided by a comparative analysis of the current level of solar activity with the reconstructed solar activity on a time scale of a few hundred or thousand years.

With the exception of occasional sightings of extremely large (naked eye) sunspots, the direct observation of sunspots has been limited to a period of about 400 years. While these early observations are incomplete, they indicate that the sunspot activity from mid-1600 till early 1700 was extremely low the period referred to as Grand, or Maunder Minimum. The reconstructions of sunspot activity obtained on the basis of visual observations span a period which started in 1610 [7, 8, 9]. This data shows a significant decrease in the amplitude of 11-year solar cycles in the second half of the 17th century, known as the Maunder Minimum (MM) 10]. In addition, based on data from optical observations, Gleissberg [13] discovered a modulation in sunspot activity with a period between 80-100 years [14] which now bears his name.

Extremely low and prolong minimum of activity between Cycles 23 and 24 , low amplitude of cycle 24, and weak polar fields at the declining phase of Cycle 24 instigated number of studies into a possibility of next Grand Minimum (GM). The predictability a GM was studied by [11] from the point of view of dynamo processes. It was found that GM could occur as the result of random fluctuations in parameters determining the behavior of dynamo, and hence, no long-term forecast of GM is possible. Several other studies, however, argued that the GM may represent the regular changes in solar activity [9, 12] and 
that the secular and bi-centennial variations may play an important role in the development of GM.

The research of long-term changes in solar variation in pre-telescoping era must be conducted using a proxy of solar activity, such as by measuring the concentration of radioisotopes $\left({ }^{14} \mathrm{C},{ }^{10} \mathrm{Be}\right)$. This data covers a time interval of several thousand years. Solar activity modulates the flow of cosmic rays, which affects the concentration of the ${ }^{14} \mathrm{C}$ in tree rings and ${ }^{10} \mathrm{Be}$ in ice cores 22. Variations in the flow of cosmic rays occur in the heliosphere, where they interact with the heliospheric magnetic field, which is a part of the larger solar magnetic field [3]. The rate of the formation of cosmogenic radionuclides is inversely proportional to solar magnetic activity. This inverse correlation is caused by the nonlinear effect of the shielding of the solar magnetic field and geomagnetic field [4].

The timespan for which there are estimates of solar activity based on radioisotopes analysis significantly exceeds that of the period for direct observations of solar activity (see [5, 21], for review). These reconstructions of solar activity indicate the presence of grand minima, similar to the Maunder minimum, as well as grand maxima, similar to the solar activity in the second half of the twentieth century. These reconstructions allow us to suggest that the sun spends approximately $15 \%$ of its life in grand minima phases, and approximately $10 \%$ in grand maxima phases [6].

Investigating the extrema of solar activity over the last 10000 years, [19] pointed out that the eras of time around $700 \mathrm{BC}$ and 450 years ago were periods of activity close to grand minimum. The paper by [15] analyzed the Fourier spectra of a combined dataset consisted of dendrochronological records, proxy of solar activity based on aurora records, and a modified sunspot number time series. During 4,500 years covered by the dataset, they noted the presence of 210 year periodicity, which was most clearly defined around the Maunder minimum. Prior and after that amplitude of 200-year periodicity was suppressed.

The main objectives of this work are to explore the timing of occurrence of Grand minima and to study their relation to 200-yearlong cycles of activity 
based on data from proxy indices as well as from direct observations of sun spots. On the basis of a comparison of phases of 200-yearlong cycles with the eras of grand minima, we also try forming a prediction about the onset of the next grand minimum of solar activity.

\section{Methodology}

The dataset used here is based on ${ }^{14} C$ radioisotope analysis. The datasets covers approximately 11 thousand years, and it was previously used by [17] as a proxy of sunspot number. This dataset was extended to more recent time using properly scaled sunspot number data. Early study by [17] found that solar activity varied greatly in this period of time, during which periods of grand solar minima, similar to the Maunder Minimum, repeatedly occurred. We use this dataset to identify key conditions for the occurrence of grand minima. Longterm changes are mainly stochastic. However, some patterns can be identified.

In the paper by [12], the authors identified certain patterns in the changing of magnetic activity between consecutive grand minima, which, as a rule, repeat after approximately every $\sim 2300$ years. Fig. 1 shows the reconstruction of sunspot quantity divided into segments of 2450 years each. These clusters are characterized by a significant rise in the level of activity from very low to very high. The clusters 3300-2860 BC and 750-350 BC were periods of great activity limited by two grand minima of activity (Fig. 1a, 1b). The duration of these clusters is about 400 years. The most recent grand minimum of activity, the Maunder Minimum, occurred about 350 years ago. Then, after 1700, there was a significant increase in activity (Fig. 1c). The growth of activity was interrupted by century minima of activity: the Dalton Minimum and the Gnevyshev Minimum in the beginning of the 20th century [18]. Century minima have a shorter duration and depth compared to grand minima (Fig. 1). Currently, after $\sim 1980$, there is a drop in activity. Fig. 1 presents the results of a reconstruction of sunspot activity in three periods, in which the level of solar activity reached a maximum of 60 units. According to [17], over the last 
7000 years, these were the moments $3155,2515,2075,435 \mathrm{BC}$, and the current era. When interpreting the timing of particular periods of past solar activity, the reader needs to remember that the accuracy and precision of radioisotope analysis depends on ability to measure precisely the isotope's fraction, which declines farther in time the measurements go. IntCal98 dataset used by [17] is provided at 10 year cadence. As a normal practice, in the range of $0-10000$ years, radiocarbon date is rounded to nearest 10 years. In the range of 1000025000 years, it is rounded to nearest 50 years. Errors in early years are higher: average standard deviation $(\sigma)$ in the range of $10000-7000$ years is about 15 years, and it is about 10 years between $7000-0$ years. By convention, zero-year corresponds to the calendar year 1950. Thus, it is not unreasonable to expect that the uncertainty in the position of peaks and troughs is about 20 years.

\section{Results}

To further explore the role of 200-year periodicity we performed the following operations with our dataset. First, we smooth the time series by 9-point (90 years) moving average, and subtract it other average formed by smoothing the dataset by 33-point moving average. This operation has a similar result as a pass-band filter, which only transmits periods between 90 and 330 years. The resulting time series is designated as $S_{200}$, and is shown in Fig. 2.

The plot shows strong quasi-periodic component, which is present over the entire time series. The main period of variations appear to be in the range of 206 and 225 years.

For the period between -2000 (2000 B.C.) and 2000 (2000 A.D.), the minimum in $S_{200}$, occurs in about 1865, close in time to the Maunder Minimum (1645-1715). The magnitude of the peak was -15.75. Assuming that a level of -15 is indicative of an onset of a grand minimum of activity, we can identify potential grand minima in the years: $-360 ;-760 ;-2870 ;-3320 ;-3935 ;-6410$; $-7020 ;-7560 ;-8210 ;-9210$.

Now, lets consider the pattern of 200 year variations in relation to these 
potential grand minima. Since we do not know the exact period of 200-year oscillations, we divided the time series on three subsets and found the harmonic oscillations with periods in about 200-year range best fitting each period (Fig. 3 ). These periods were found to be about $T \sim 205$ years(for time interval between -10000) and -6000 years), $T \sim 215(-6000--2000)$ and $T \sim 204$ ($2000-+2000)$. With the exception of GM9, all other potential Grand Minima occurred around the troughs of 200-year periodic variation. Fig. 3c also shows three known minima in sunspot activity: the Oort, Wolf, and Spörer minima, which by amplitude are smaller than the Grand (Maunder) Minima. The timing of these minima also close to troughs of 200 -year periodicity. The reader may also notice that the Dalton minimum does not appear in $S_{200}$ series.

While the significant minima in sunspot activity seem to occur near the troughs of 200-year periodicity, not all such minima are deep enough to become "Grand Minima". As an additional clue, we note that the grand minima identified by our threshold in $S_{200}$ series, seem to occur in clusters (see, Fig. 1). Extending this analogy to most recent of Grand Minima, the Maunder Minimum, allows us making a prediction that the next grand minimum could be expected to occur around $\sim 2090 \pm 20$. Here is the uncertainty is based on general properties of the dataset as it was briefly discussed in section 2 (Methodology). The reader may also note that the secondary peaks in the clusters $(-760)-(-360)$ and $(-3935)-(-2870)$ were deeper than the first ones (Fig. 1). Thus, one can speculate that the minimum in $\sim 2090$ could also be deeper than the Maunder minimum.

\section{Discussion}

One important outcome of this work is the conclusion that the great minima form clusters. In Fig. 2 and Fig.4 (upper-left) the periods of Grand Minima (GM) can be identified by larger amplitude in $S_{200}$ variation and smaller variances (Fig. 4, upper-right). This agrees with [15], who found that 200-year periodicities strengthen during GM epochs. Fig. 4 shows a wavelet analy- 
sis of $S_{200}$ index. The amplitude of the index increased around $\sim-9000$ : $-7000 ;-3330,-700$ (negative years correspond to years prior to 1950) and in the current era. These clusters are also identifiable in the wavelet power spectrum (Fig. 4, low-left, areas colored by dark red. The periods "hosting" clusters of Grand Minima are separated by periods of higher activity. The length of these periods is about 2000 years. This seems to be in a qualitative agreement with [12], who suggested that the Grand Minima may occur with a periodicity of $\sim 2300$ years.

Could the clustering patterns appear by chance? To investigate that we created two artificial datasets: D1 and D2 using the original data set from [17] only. First, we subtracted the running mean (100 points) from this dataset, and analyzed the residuals. The distribution of residuals was found to correspond well to the normal (Gaussian) distribution, with zero mean. To create D1 dataset, we used the parameters of fitted Gaussian to create a random noise component and added this noise to (previously subtracted) running mean component found from the observations. This artificial dataset was analyzed using the same approach as in $S_{200}$ analysis discussed in Section 3. To demonstrate the similarity in the distribution of residuals in observed data set and those of random noise, Fig. 5 compares the two distributions. Next we applied our band pass filter (see Section 2) to new datasets, and the results are shown in Fig.6. It is clear that while there are similar variations in both observed and artificial datasets, in the artificial data the deviations are not different between the location of possible Grand Minima (GM) and other locations. None of those are too low to cross the level of -15 .

Second artificial data set (D2) is similar to D1, except the random component was created by randomly reordering the actual (observed) residuals. For statistical analysis, we run the independent realizations of D1 and D2 10,000 times. Out of 10,000 realizations, the $S_{200}$ index contained at least one peak below -15 threshold in 847 cases $(8.47 \%$, for D1) and 1054 cases $(10.54 \%$, for D2). There were only $10(0.1 \%, \mathrm{D} 1)$ and $21(0.21 \%, \mathrm{D} 2)$ cases when the resulting time series produced 2 peaks below the "Grand Minima" threshold. Number of 
3-peak cases was even smaller ( 1 for D1 and 2 for D2). This statistics refers to the total number of minima below -15 threshold. There were no cases when the low peaks appeared in groups of two or more. Based on this statistical case, it appears that while it is possible to get a peak in $S_{200}$ time series bellow -15 from a purely random distribution, the number of such peaks is significantly smaller than in the observed time series, and none of the peaks appear in pairs or groups. From statistical perspective, we shall conclude that the patterns of low peaks we find in $S_{200}$ index do not appear by chance.

Several methods have been developed historically to convert measured cosmogenic isotope data into a solar activity index, ranging from very simple regressions to physics-based models. A new step in long-term solar-activity reconstruction has been made recently, which is the development of the proxy method in which physics-based models are used, instead of a phenomenological regression, to link sunspot number (SN) with cosmogenic-isotope production [17, 22, 23, 20]. Some may argue that the ${ }^{14} \mathrm{C}$ dataset previously used by [17] is based on outdated SN timeseries for interpreting the radioisotopic measurements in the framework of solar open magnetic field.To rebut this suggestion, we added a reconstruction [22] for comparison. The series [22] use multi-proxy approach based on different cosmogenic proxy data combined in a joint reconstruction. $S_{200}$ index derived from [22] dataset is shown in Fig. 2. Both time series yield similar results.

\section{Conclusion}

In this study, we performed an analysis on the long-term reconstruction of solar activity. We introduced a new index, $S_{200}$, which characterizes the 200 year periodicity of solar activity, which we got by anti-aliasing 11-year cycles and by subtracting trends with a period of more than 330 years. We clarified the time of onset of grand minima over the last 11000 years. We have 
identified 11 such minima. These minima occurred in $1683 \mathrm{AD}$ and 360; 760; $2870 ; 3320 ; 3935 ; 6410 ; 7020 ; 7560 ; 8210 ; 9210$ BC. We showed that the last grand minima occurred in pairs in time intervals of 400-600 years and happened in the minima era of quasi-harmonic modulation of index $S_{200}$. Famous solar activity minima Oort,Wolf, Spörer and Maunder minima, which occurred during the local minima of a $\sim 200$-year cycle. Variations 200 year period may have not only statistically but physical nature. So there is evidence that in certain phases of the 200-year cycle is reversal Gnevyshev-Ohl rule for even and odd cycles. The $S_{200}$ series shows a clustering of Grand Minima. Extending these findings to future solar activity, we expect that the middle of the next grand minimum will occur in approximately 2090. Based on a pattern found in past GM, it is possible that the amplitude of next GM minimum will be deeper than that of the Maunder Minimum.

\section{Acknowledgments}

The work by A. Tlatov was partially supported by the Russian Science Foundation, grant 15-12-20001. Authors also indebted to Irina Plaks for her assistance in preparing the English version of the manuscript. A.A.P. acknowledges the financial support by the Academy of Finland to the ReSoLVE Centre of Excellence (project No. 272157). The National Solar Observatory (NSO) is operated by the Association of Universities for Research in Astronomy, AURA Inc under cooperative agreement with the National Science Foundation (NSF).

\section{References}

[1] Vitinsky Yu. I., Kopecky M., Kuklin G. V., The statistics of sunspots (Statistika pjatnoobrazovatelnoj dejatelnosti solntsa). Moscow: Nauka, 1986.

[2] Dunai T. J. Cosmogenic Nuclides: Principles, Concepts and Applications in the Earth Surface Sciences. Cambridge University Press, Cambridge, 2010. 
[3] Lean J. L., Wang Y.-M., Sheeley N. R. The effect of increasing solar activity on the Sun's total and open magnetic flux during multiple cycles: Implications for solar forcing of climate. Geophys. Res. Lett., 2002; 29:2224.

[4] Lal D., Peters B. Cosmic Ray Produced Radioactivity on the Earth. In: Kosmische Strahlung II / Cosmic Rays II , Sittle K. ed., Handbuch der Physik / Encyclopedia of Physics, 9/46/2, 612, Springer, Berlin; 1967.

[5] Usoskin I. G. A History of Solar Activity over Millennia, Living Rev. Solar Phys., 2013; 10.

[6] Usoskin, I. G., Solanki, S. K., Kovaltsov, G. A. Grand minima and maxima of solar activity: new observational constraints. Astron. Astrophys., 2007; 471:301-9.

[7] Hoyt D. V., Schatten H. Group Sunspot Numbers: A New Solar Activity Reconstruction. Solar Phys., 1998; 181:189-20.

[8] Clette F., Svalgaard L., Vaquero J. M., Cliver E. W. Revisiting the sunspot number. a 400-year perspective on the solar cycle. Space Science Reviews. 2014; 186: 35-103.

[9] Zolotova, N.V., Ponyavin D.I., How Deep Was the Maunder Minimum? Solar Phys., 2016, 291:2869-2890.

[10] Eddy J. A. The Maunder Minimum, Science, 1976; 192:1189-14.

[11] Usoskin I. G., Sokoloff, D., Moss D., Grand Minima of Solar Activity and the Mean-Field Dynamo. Solar Phys. 2009; 254: 345-355.

[12] Mordvinov A. V., Kramynin A. P., Long-Term Changes in Sunspot Activity, Occurrence of Grand Minima, and Their Future Tendencies, Solar. Phys., $2010 ; 264-5$.

[13] Gleissberg W. A long-periodic fluctuation of the sun-spot numbers. Observatory, 1939; 62:158-2. 
[14] Rozelot J. P. On the stability of the 11-year solar cycle period (and a few others), Solar Phys., 1994; 149:149-6.

[15] Bonev B. P., Penev K. M., Sello S. Long-Term Solar Variability and the Solar Cycle in the 21st Century [letter]., ApJ 2004; 605:81-4.

[16] Inceoglu F., Simoniello R., Knudsen M. F., Karoff C., Olsen J., TurckChièze, S. On the Current Solar Magnetic Activity in the Light of Its Behaviour During the Holocene. Solar. Phys., 2015; 291-12.

[17] Solanki S. K., Usoskin I. G., Kromer, B., Sch`ussler, M., Beer, J. Unusual activity of the Sun during the previous 11,000 years. Nature, 2004; 431:1084-2.

[18] Usoskin I.G., A History of Solar Activity over Millennia. Living Rev. Solar Phys., 2008; 5:3.

[19] Dergachev V. A. Radiocarbon chronometer., Priroda, 1994; 1: 3-12.

[20] Usoskin I.G., Gallet Y., Lopes F., Kovaltsov G.A., Hulot G. Solar activity during the Holocene: the Hallstatt cycle and its consequence for grandminima and maxim. Astron Astrophys, 2016; 587:A150. doi:10.1051/0004$6361 / 201527295$

[21] Usoskin I.G., A history of solar activity over millennia. Living Rev. Solar Phys., 2017; 14:3.

[22] Steinhilber F., Abreu J.A., Beer J., Brunner I.,Christl M., Fischer H.,Heikkila U.,Kubik P.W.,Mann M., McCracken K.G., Miller H., Miyahara H., Oerter H., Wilhelms F., 9,400 years of cosmic radiation and solar activity from ice cores and tree rings. Proc Natl Acad Sci USA, 2012; 109(16):59675971. doi:10.1073/pnas. 1118965109

[23] Muscheler R., Adolphi F., Herbst K., Nilsson A. The Revised Sunspot Record in Comparison to Cosmogenic Radionuclide-Based Solar Activity Reconstructions, Solar Phys., 2016, 291: 3025 
Figure 1: Reconstruction of the sunspot index for the periods: (a) 4955-2550 BC (b) 2545-105 $\mathrm{BC}$, (c) $100 \mathrm{BC}$ - present time according to [17]. Marked are the positions of the latest minima of activity. Shaded in are the characteristic clusters of heightened activity, between successive grand minima. 


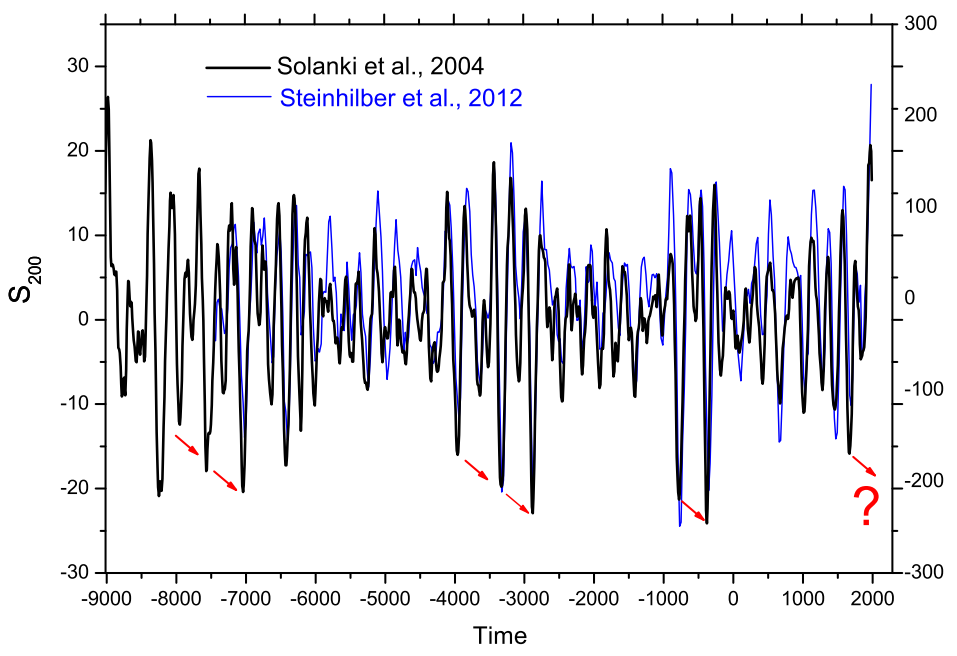

Figure 2: Variations in solar activity, found by subtracting series which were obtained from radioisotope analysis, smoothed by 90 years and 330 years. For comparison, two independent reconstructions based on data from [17] and [22] are shown. 

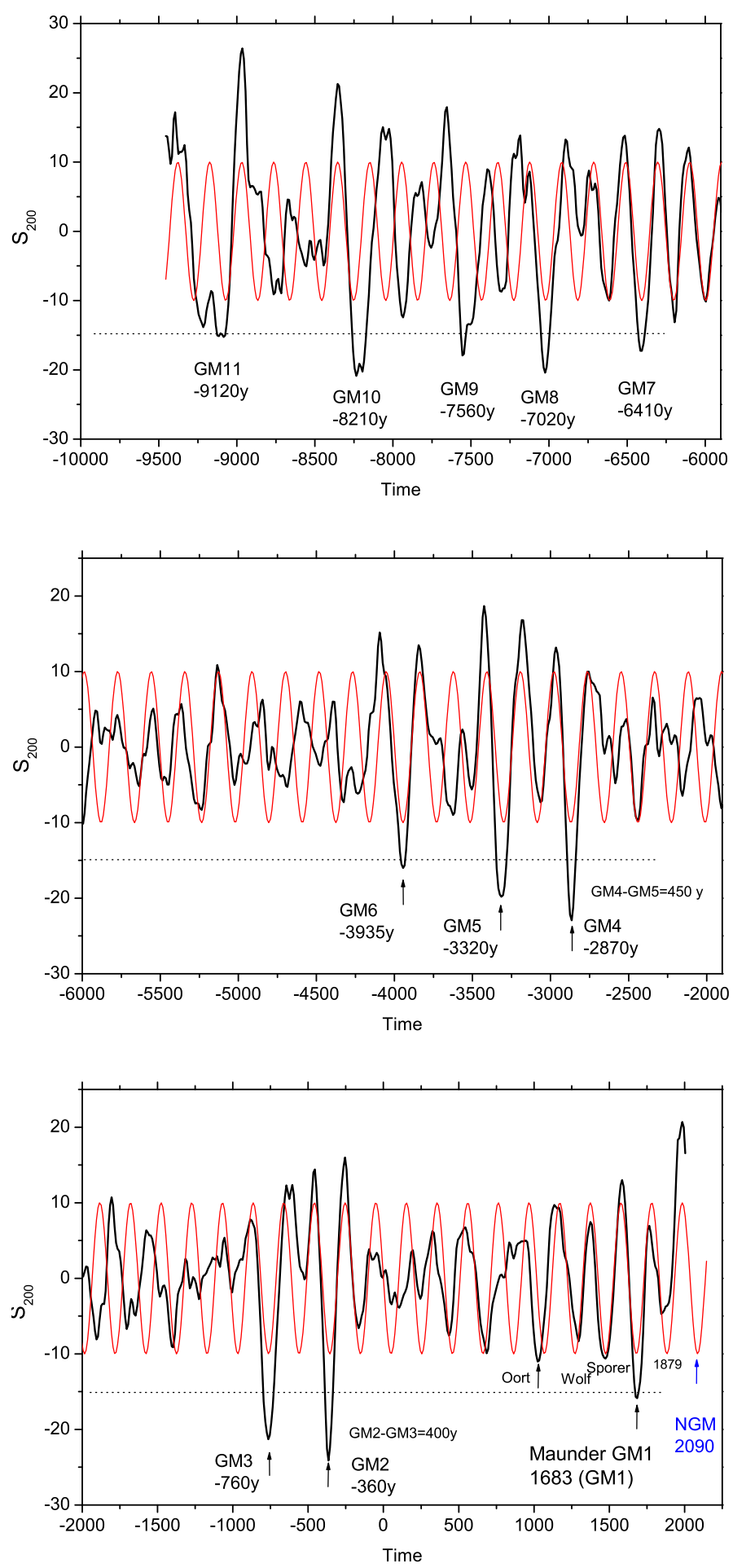

14

Figure 3: More detail than Fig.2 from data set [17]. Also added were harmonic oscillations with a 204 year period for the time interval $-2000 \div 2000$ yrs., a 215 year period for the time interval -6000-2000, and 205 year period for the timer interval before -6000 . A harmonic sinusoidal curve plotted in red is shown for a reference only. It does not represent any fitting to the observed data. 

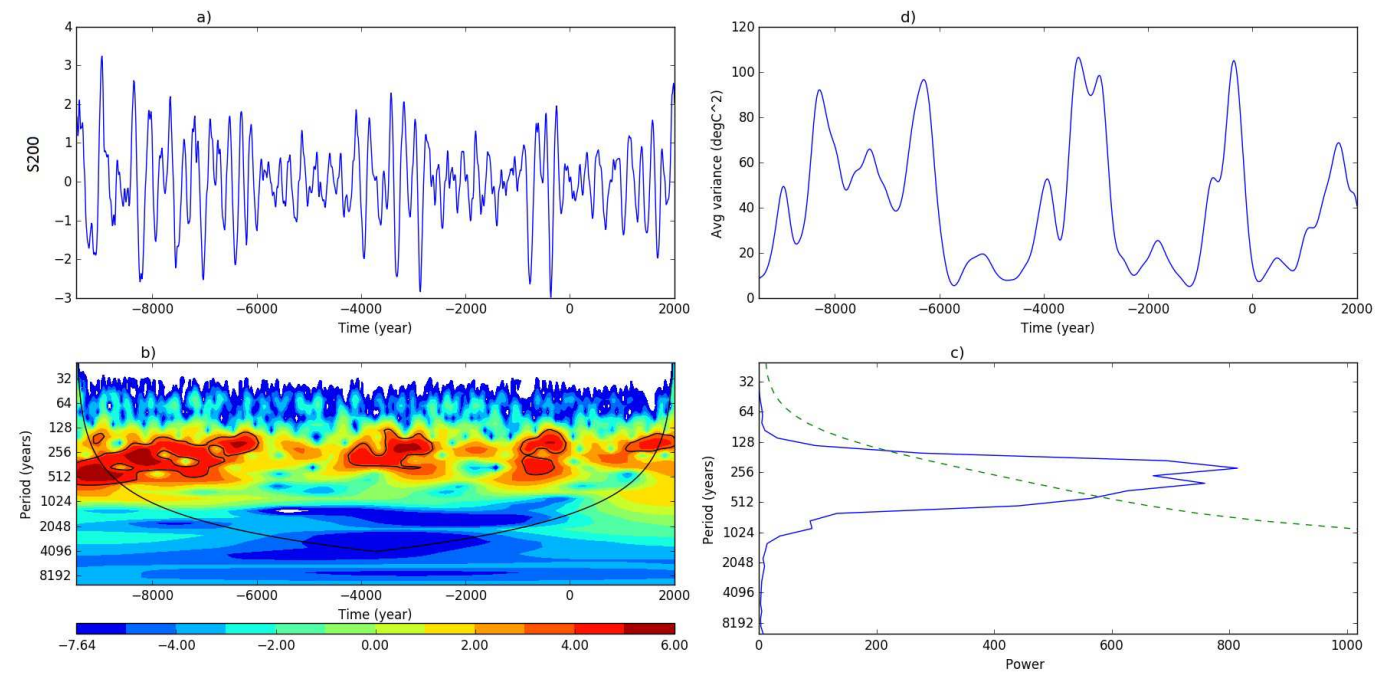

Figure 4: Spectral analysis of 200 year oscillations. a) Time series S200; b) Wavelet power spectrum; c) Scale average time series for periods 100-300 years; d) global wavelet spectrum. 


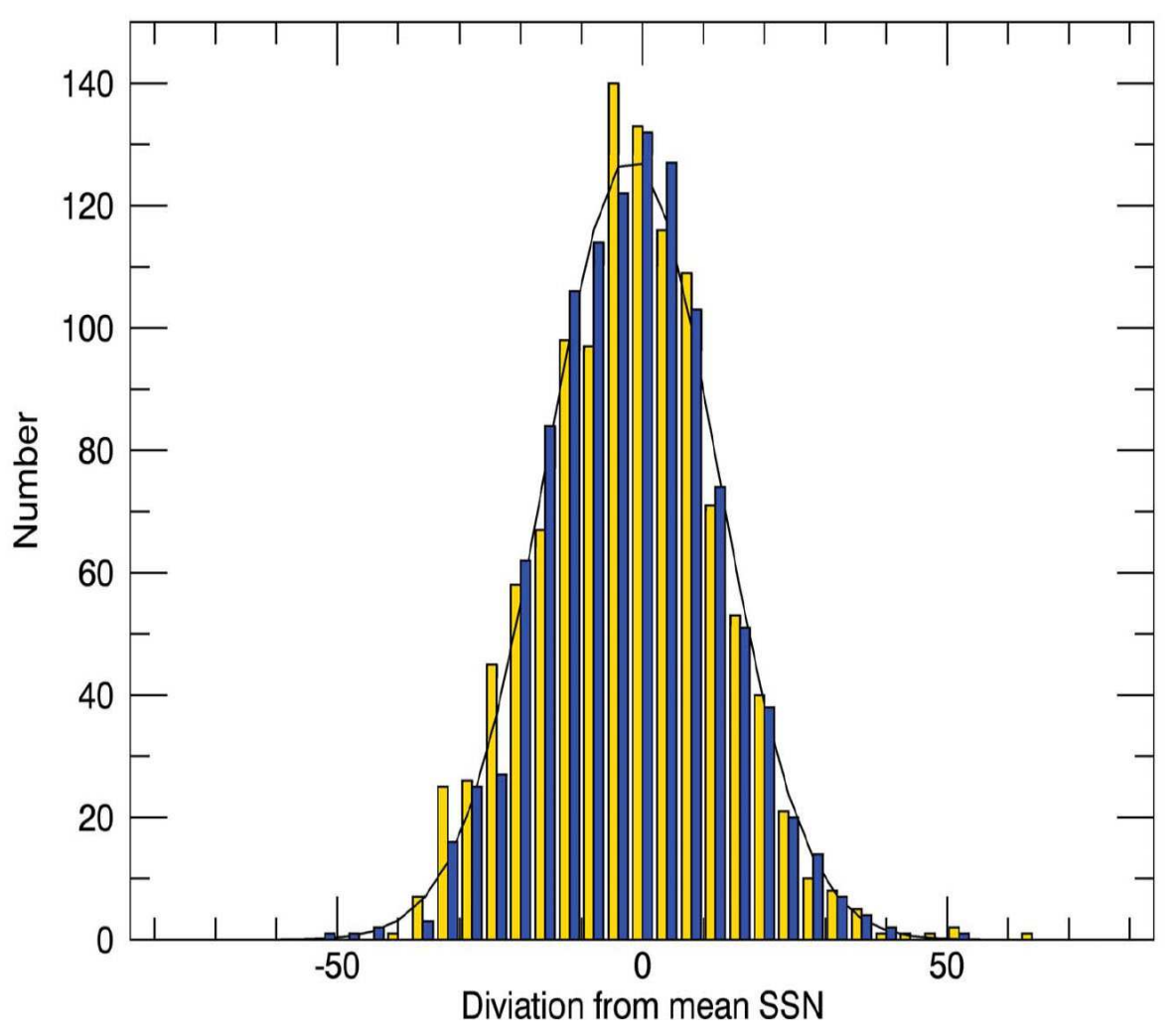

Figure 5: The deviations data from data set [17] after subtracted 100 points running mean. The deviations seems to be fitted well by the Gaussian function (yellow bars, black line). Blue bars on plot show that noise the deviations from running mean have the same statistical properties as the deviations in observations. 


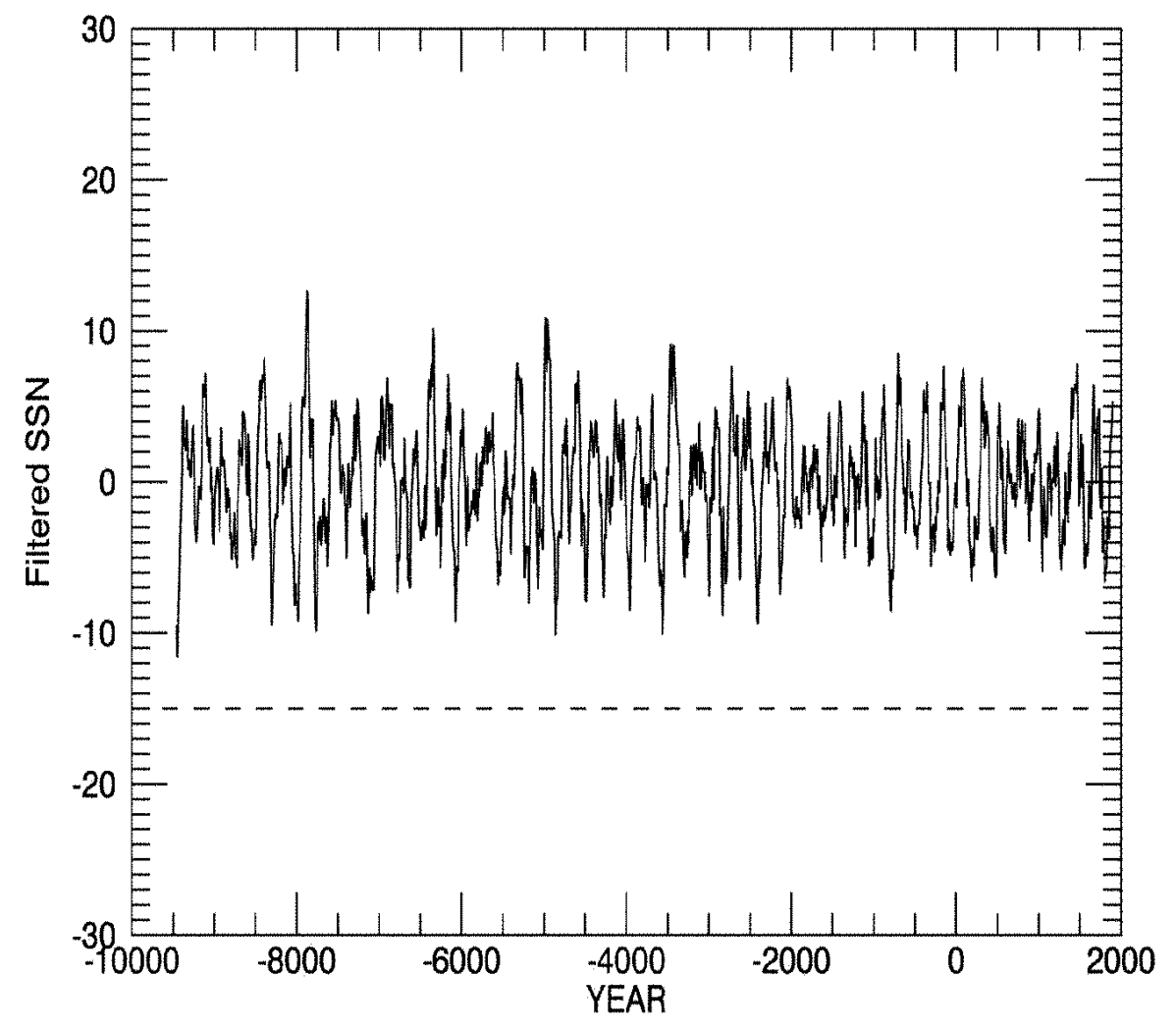

Figure 6: The artificial dataset the created from series 17], in which Gaussian noise imposed (Fig. 5), and applied filtering (see Section 2). In this series, there are no deviations below -15 . 